\title{
Optimization of production of the fermented milk beverage based on Tibetan kefir grains
}

\author{
Rustem Khabibullin ${ }^{1, *}$, Mahmud Adylov ${ }^{1}$, and Galina Ezhkova ${ }^{1}$ \\ ${ }^{1}$ Kazan National Research Technological University, K. Marks St. 68, Kazan, 420015, Russian Federation
}

\begin{abstract}
In recent years we have seen interest in Tibetan kefir grains (TKG) for production of fermented milk beverages. The article presents the results of the work on expanding the range of fermented milk beverages based on TKG, studying the influence of fermentation conditions on quality characteristics of beverages and their shelf life. The possibility of their optimization is shown. Using the method of Central Composite Design (CCD), response functions were obtained in the form of 2nd-order polynomials, and the numerical values of the coefficient of these dependencies were calculated. The conditions of milk fermentation by Tibetan kefir grains were optimized. The numerical values of the influencing factors are: the dose of inoculate is $(60 \pm 2) \mathrm{g}$ per $100 \mathrm{ml}$ of the milk, fermentation duration - $(30 \pm 3) \mathrm{h}$; fermentation temperature $-(35 \pm 4)^{\circ} \mathrm{C}$. We also studied the effect of sea buckthorn, chokeberry and lingonberry juice additives on the quality of fermented milk drinks and their shelf life. Using the same method CCD we determined the functional dependencies of sensory indicators and shelf life of the beverage on the added amount of berry juice and sucrose. According to the results of the conducted studies, the recipe of a fermented milk beverage was modified. The optimal content of the components (per $100 \mathrm{ml}$ of the drink) is: juice $-(12 \pm 2) \mathrm{ml}$, sucrose - $(10 \pm 1) \mathrm{g}$.
\end{abstract}

\section{Introduction}

Fermentation processes used to be used only to increase shelf life of products and improve their taste, but in recent years fermented foods have become associated with a healthy diet and have attracted scientific interest [1]. This role of fermented foods brought to life a new class of foods called functional nutrition [2].

The microorganisms that provide fermentation process are also becoming the object of study, because they have a wide range of biological activity: they control the growth of probiotic microorganisms, synthesize vitamins, specific proteins, highly digestible calcium, bacteriocins, sphingolipids and many others. As a result, fermented foods provide many health benefits such as anti-oxidant, anti-microbial, anti-fungal, antiinflammatory, anti-diabetic, anti-carcinogenic activity [1], prevention of gastrointestinal infections, lowering serum cholesterol and antimutagenic activity. Fermented foods are recommended for people with lactose intolerance and atherosclerosis [3]. L. Acidophilus and Bifidobacterium spp. are also known for their probiotic properties, but fermented foods should contain them in the amount of at least $10^{6} \mathrm{cfu} / \mathrm{g}$ [4].

At the end of the millennium hypotensive effect of fermented products due to biologically active peptides was discovered. The peptides are released during fermentation or gastrointestinal digestion. Such fermented dairy products exist, they are few in number, and are based mainly on Lactobacillus helveticus [5-10].
A well-known kefir is a traditional Middle Eastern fermented milk drink. It is a product of fermentation of milk with kefir grains or mother cultures made from grains. It consists of various species of Lactobacilli, Lactococci, Leuconostoc, Acetobacter and yeasts (both lactose- and galactose-fermenting). Kefir has a fizzy, sour taste, tart and refreshing aroma, it contains essential amino acids, and has healing and supporting functions [11]. Some products, such as yogurt, yogurt powder, and cottage cheese powder (dahi), are very popular and produced [3].

In recent years we have seen interest in Tibetan kefir grains (TKG) for production of fermented milk beverages. The microbial composition of TKG varies depending on its origin and substrate.

Its microbial composition differs from Russian kefir, Irish kefir, Taiwanese kefir, Turkish fermented drink with kefir. Tibetan kefir consists also of Lactobacilli (Lactobacillus casei, Lactobacillus hilgardii, Lactobacillus delbrueckii spp., Lactobacillus plantarum, Lactobacillus kefir, Leuconostoc mesenteroides spp. dextranicum), Lactococci (Streptococcus lactis), yeast (Zygosaccharomyces cerevisiae, Torulospora pretoriensis, Candida lambica, Candida valida), and a small amount of acetic bacteria.

The special grain structure is due to the ability of the bacteria Lactobacillus kefiranofaciens and the yeast Saccharomyces turicensis to self-aggregate into small granules. The biofilms-producers Lactobacillus kefiri, Kluyveromyces marxianus and Pichia fermentans adhere

\footnotetext{
* Corresponding author: hrustik@yandex.ru
} 
to the granules surface, which turns them into kefir grains. This process is facilitated by the symbiotic relationship between bacteria and yeasts of kefir grains. Later, Lactobacillus kefiri, L. kefiranofaciens, $L$. kefirgranum, Lactobacillus parakefir, and Candida kefir were classified using the product name [12].

The high antioxidant potential (AP) of dairy products allows us to overcome the negative effects of oxidative stress. This high AP is explained by the breakdown of milk protein during fermentation or digestion and the release of antioxidant peptides, which increase AP by 25 times. Fat milk has higher AP than low-fat and skim milk due to lipophilic antioxidants, and Lactobacillus casei/acidophilus provides the highest AP among tested in the LAB [13].

Lactobacillus plantarum MA2 strains isolated from Chinese TKG have high antioxidant activity in vitro and in vivo, acid and bile salt tolerance, cell surface hydrophobicity and auto-aggregation, which confirmed the probiotic characteristics of these bacteria of TKG [14]. The high cellular antioxidant activity (CAA) has been studied both at different stages of cell growth and for different components of fermentation media - cellfree extract and fermentation supernatant, and CAA method was recommended to use for lactic acid bacteria antioxidant potential evaluation [15]. It was shown that the level of total cholesterol, triglycerides, and lowdensity lipoproteins in the blood serum of rats, which received a certain amount of $\mathrm{LAB}$ strains isolated from TKG, reduced significantly. It was recommended to use these strains as probiotic starter cultures for production of fermented products [9]. Similar results were obtained for other microbial strains from TKG [16].

Some components synthesized by microbial strains from TKG also have antioxidant and gut microbiotaregulating activity. For example, exopolysaccharide (EPS) produced by Lactobacillus plantarum YW11 reduced oxidative stress in aging mice with increased levels of glutathione peroxidase, superoxide dismutase, catalase and total serum antioxidant capacity. Moreover, EPS can restore the diversity of the microbiota of experimental animals. The fermented milk drink containing EPS also showed a beneficial antioxidant effect [17].

It was found that different substances with antifungal activity are formed during different stages of TKG microbial community cultivation. Starting from $36 \mathrm{~h}$ of fermentation antifungal activity is connected mainly with sensitive to heat peptides and proteolytic enzymes. Starting from 3-4 days of cultivation and later organic acids and/or substances active in acidic environment are accumulated. At a later stage of cultivation, a certain contribution to the antifungal effect is also made by thermally-stable, protease-resistant substances [18].

It was shown that TKG can be used for other dairy products manufacturing, for example, Camembert type cheese $[19,20]$, and traditional Chinese Bod ljong cheese [21]. The fermenting activity of TKG has been shown not only for milk, but also for a mixture of mulberry juice and whey [22].

Several key factors, which determine the probiotics ability to survive in the product and human gastrointestinal tract, should be taken into account. These factors include physiological state of the introduced probiotics, storage conditions of a product, its chemical composition, including acidity, carbohydrate content, nitrogen sources, mineral content, water activity and oxygen content, and possible interactions between probiotics and starter cultures [23]. It was shown that Lactobacillus acidophilus and Bifidobacterium animalis subsp. lactis ratio strongly affects the properties of a fermented milk product, including acidification profile, organic acid production, lactose consumption, shelf life at $4^{\circ} \mathrm{C}$, bacterial viability, sensory properties, organic acid content and viability under gastrointestinal conditions [4]. Another paper describes the optimizing method of the TKG culture growing using the response surface analyzing. As a result, the optimal values of skimmed milk quantity, temperature, time of fermentation and inoculate amount were determined [24].

The enrichment of dairy products with natural ingredients, containing a considerable amount of essential nutrients, is used to develop the technology of fermented milk products. Spirulina, chickpea, St. John's wort, tarragon, mint, sage, stevia, chili pepper, black pepper, cinnamon, allspice, thyme, cumin and many others are used as additives [25-29]. Use of these spicy and aromatic additives improves and diversifies the taste and aromatic properties of fermented products, enrich them with biologically active substances, increase their stability when stored.

Given the above, broadening the range of fermented milk drinks based on the TKG, studying of the influence of fermentation conditions, formation of the characteristics of fermented milk beverages, their changes during the storage and optimization of these factors should be recognized as important areas of research.

Therefore, this paper aims:

To obtain the second-order polynomial response surfaces both of quality and shelf life indicators on the main fermentation parameters using Central Composite Design (CCD);

To optimize the resulting polynomial responses, i.e. to determine conditions providing the extremal (maximal) level of these functions;

To study the effect of plant additives on the quality of fermented beverages and their shelf life by obtaining corresponding response surfaces;

To optimize beverage formulation to provide maximum of these function.

\section{Materials and methods}

Experimental studies were conducted in laboratory of the Meat and Dairy Products Technologies Department at Kazan National Research Technological University using generally accepted methods [30] and equipment.

The following materials and objects were used and studied. Pasteurized milk with $3.2 \%$ fat content TM "Very important cow" (Zelenodolsk Dairy Plant, Republic of Tatarstan, Russia); Certified Organic Live 
Milk Kefir Grains Tibetan Starter by Kombucha Organic $\AA$; fresh sea buckthorn, chokeberry and lingonberry. Juices were prepared from berries by grinding in a blender, followed by sedimentation in a laboratory ELMI CM-50 centrifuge, Latvia.

The Tibetan kefir grains were preserved in pasteurized milk at $4^{\circ} \mathrm{C}$, and activated at $28^{\circ} \mathrm{C}$ for $24 \mathrm{~h}$. A day later the fermented product was poured into a container through a plastic sieve, while being gently stirred, separated kefir grains were washed under a stream of cold water so that they were completely clean for the next fermentation. Dishes used were thoroughly washed with water, and it was forbidden to use synthetic detergents.

The key analytical techniques used were titrimetric method according to GOST 3624-67 (acidity determination); butyrometric method according to GOST 3622-67 (weight fraction of fat); Kjeldahl method according to GOST R ISO 2446-2011 (weight fraction of protein). In some experimental series physic-chemical parameters such as fat content, protein, lactose, mass fraction of dry milk residue, skim milk residue, homogenization degree and freezing point were determined on the "Clover-2M" milk analyzer.

The titratable acidity and $\mathrm{pH}$ values were measured during the propagation. The $\mathrm{pH}$ value was determined by using a digital desktop $\mathrm{pH}$ meter Hanna Instruments HI 2216.

Descriptive sensory analysis was conducted with the participation of 5 qualified trained evaluators using the consensus method in accordance with the relevant standards (ISO 13299:2003; ISO 11035:1994; ISO 4121:2003, ISO 8587:2006) and was used as a tool for evaluating and comparing the quality of fermented beverages with berry juices. The score was given on a 9point scale.

In order to optimize the fermentation process, we applied the response surface analysis methodology (RSM) [24, 31] that provides for the Central Composite Design (CCD). As a result the second-order dependence of process efficiency indicators on the influencing factors was obtained in the following form:

$$
y\left(x_{1}, \ldots, x_{k}\right)=\beta_{0}+\sum_{i=1}^{3} \beta_{i} \cdot x_{i}+\sum_{i=1}^{3} \beta_{i i} \cdot x_{i}^{2}+\sum_{i=1}^{2} \sum_{j=i+1}^{3} \beta_{i j} x_{i} x_{j}
$$

where $\mathrm{Y}$ is a dependent variable (process efficiency or product quality indicators); $\beta_{0}, \beta_{\mathrm{i}}, \beta_{\mathrm{ii}}, \beta_{\mathrm{ij}}$ are the regression equation coefficients; $\mathrm{x}_{\mathrm{i}}, \mathrm{x}_{\mathrm{j}}$ are the levels of the influencing factors. The total number of experiments $\mathrm{N}$ was calculated using the formula:

$$
N=N_{0}+2 \cdot k+n_{0}
$$

where $\mathrm{n}_{0}$ is the number of experiments in the center of the plan, $\mathrm{k}$ is the number of factors, and $\mathrm{N}_{0}$ is the number of experiments in the full factor experiment. The value of the distance to the axial point, also called the star point, $\alpha$ is calculated by the formula:

$$
\alpha=\sqrt{\frac{\sqrt{N \cdot N_{0}}-N_{0}}{2}}
$$

The experimental data were expressed as mean \pm standard deviation (SD) from three replicates for each sample. The analysis of variance (ANOVA) of the data was performed using the Statistica 6.0 software package.

\section{Results and discussion}

\subsection{Constructing Central Composite Design}

Fermentation conditions whose influence was evaluated and selected for consideration in the experiment were the following: the inoculate (TKG) amount $(25-75 \mathrm{~g} / 100 \mathrm{~g}$ of milk), fermentation temperature $\left(20-45^{\circ} \mathrm{C}\right)$, fermentation time $(8-40 \mathrm{~h})$. We have constructed a central composite plan for three variables on 5 levels, so in accordance with formulas (2) and (3), $\alpha=\sqrt{3}=1.73$, $\mathrm{N}=17$. The levels of these factors according to CCD are shown in Table 1.

Table 1. Range of CCD values for fermentation conditions optimization.

\begin{tabular}{|c|c|c|c|}
\hline Levels & Factor 1 & Factor 2 & Factor 3 \\
\cline { 2 - 4 } & $\begin{array}{c}\text { TGK amount, } \\
\text { g/100 g of } \\
\text { milk }\end{array}$ & $\begin{array}{c}\text { Fermentation } \\
\text { temperature, } \\
{ }^{\circ} \mathrm{C}\end{array}$ & $\begin{array}{c}\text { Fermentation } \\
\text { time, } \mathrm{h}^{\circ} \mathrm{C}\end{array}$ \\
\hline-1.73 & 20 & 15 & 8 \\
\hline-1 & 25 & 20 & 12 \\
\hline 0 & 50 & 32.5 & 24 \\
\hline 1 & 75 & 45 & 36 \\
\hline 1.73 & 80 & 50 & 40 \\
\hline
\end{tabular}

The effect of the components on the sensory indicators of fermented beverages was evaluated and the amount of berry juice (5-15 g/100 g of the beverage) and sucrose (5-15 g/100 $\mathrm{g}$ of the beverage) was selected (Table 2). According to formulas (2) and (3), in this case $\mathrm{k}=2, \alpha=\sqrt{2}=1.44, \mathrm{~N}=9$.

Table 2. Range of CCD values for optimal formulation of beverages with berry juice ( 2 factors, 5 levels).

\begin{tabular}{|c|c|c|}
\hline Levels & Factor 1 & Factor 2 \\
\cline { 2 - 3 } & $\begin{array}{c}\text { Berry juice amount, } \\
\text { g/100 g of beverage }\end{array}$ & $\begin{array}{c}\text { Sucrose amount, } \\
\mathrm{g} / 100 \mathrm{~g} \text { of beverage }\end{array}$ \\
\hline-1.44 & 2.5 & 2.5 \\
\hline-1 & 5 & 5 \\
\hline 0 & 10 & 10 \\
\hline 1 & 15 & 15 \\
\hline 1.44 & 17.5 & 17.5 \\
\hline
\end{tabular}

As a response function, a comprehensive quality criterion (CQC) was used, including taste, color, smell, and consistency.

$$
\mathrm{CQC}=(\mathrm{T}+\mathrm{Cl}+\mathrm{S}+\mathrm{Cn}) / 4,
$$

where $\mathrm{T}$ - evaluation of taste, $\mathrm{Cl}$ - rating of color, $\mathrm{S}$ score of smell, Cn - assessment of consistency, 4 number of indicators used. 
As the second response function, the product shelf life index was used, which was determined by the dynamics of the $\mathrm{pH}$ value changes during product storage at a temperature of $+18^{\circ} \mathrm{C}$. The time when the $\mathrm{pH}$ changes to an upward trend, is rounded to 12 hours and expressed in days, and is considered the storage time.

\subsection{Optimization of fermentation conditions}

In order to optimize the process of milk fermentation by TKG, a graph of the dependence of the process efficiency indicators on the influencing independent parameters (factors) was obtained based on the results of the laboratory studies. The main parameters which influence the quality indicators of the fermented milk were varied: the amount of inoculate (introduced starter culture) from 25 to $75 \mathrm{~g} / \mathrm{l}$; fermentation time from 12 to 36 hours; fermentation temperature from 20 to $45^{\circ} \mathrm{C}$. The Central Composite matrix is presented in Table 3.

Table 3. Matrix of the central composite design for optimizing fermentation conditions.

\begin{tabular}{|c|c|c|c|}
\hline \multirow{2}{*}{$№$} & \multicolumn{3}{|c|}{ The levels of factors } \\
\cline { 2 - 4 } & $\begin{array}{c}\text { Factor } \mathrm{X}_{1} \\
\text { Inoculate } \\
\text { amount, } \mathrm{g} / \mathrm{l}\end{array}$ & $\begin{array}{c}\text { Factor } \mathrm{X}_{2} \\
\text { Fermentation } \\
\text { time, } \mathrm{h}\end{array}$ & $\begin{array}{c}\text { Factor } \mathrm{X}_{3} \\
\text { Fermentation } \\
\text { temperature, }{ }^{\circ} \mathrm{C}\end{array}$ \\
\hline 1 & 75 & 36 & 45 \\
\hline 2 & 25 & 36 & 45 \\
\hline 3 & 75 & 12 & 45 \\
\hline 4 & 25 & 12 & 45 \\
\hline 5 & 75 & 36 & 20 \\
\hline 6 & 25 & 36 & 20 \\
\hline 7 & 75 & 12 & 20 \\
\hline 8 & 25 & 12 & 20 \\
\hline 9 & 80 & 24 & 32.5 \\
\hline 10 & 20 & 24 & 32.5 \\
\hline 11 & 50 & 40 & 32.5 \\
\hline 12 & 50 & 8 & 32.5 \\
\hline 13 & 50 & 24 & 15 \\
\hline 14 & 50 & 24 & 50 \\
\hline 15 & 50 & 24 & 32.5 \\
\hline 16 & 50 & 24 & 32.5 \\
\hline 17 & 50 & 24 & 32.5 \\
\hline
\end{tabular}

The values of sensory indicators $\mathrm{T}, \mathrm{Cl}, \mathrm{S}, \mathrm{Cn}$, summarized as CQC, as well as the shelf life are presented in Table 4.
Table 4. Sensory indicators, CQC and shelf life of fermented milk.

\begin{tabular}{|l|l|l|l|l|l|l|}
\hline № & \multicolumn{1}{|c|}{$\mathrm{T}$} & \multicolumn{1}{|c|}{$\mathrm{S}$} & \multicolumn{1}{|c|}{$\mathrm{Cl}$} & \multicolumn{1}{|c|}{$\mathrm{Cn}$} & \multicolumn{1}{|c|}{$\mathrm{Y}_{1}$} & $\mathrm{Y}_{2}$ \\
\hline 1 & 8.5 & 7.5 & 8.0 & 8.0 & 8.0 & 7.0 \\
\hline 2 & 8.0 & 7.0 & 7.0 & 6.0 & 7.0 & 6.0 \\
\hline 3 & 8.0 & 7.5 & 7.5 & 8.0 & 7.7 & 6.5 \\
\hline 4 & 7.0 & 7.0 & 7.5 & 7.5 & 7.2 & 5.5 \\
\hline 5 & 8.5 & 8.0 & 8.0 & 8.0 & 8.1 & 7.0 \\
\hline 6 & 7.5 & 7.0 & 7.0 & 7.5 & 7.25 & 6.0 \\
\hline 7 & 8.0 & 7.0 & 8.0 & 8.0 & 7.75 & 6.5 \\
\hline 8 & 7.0 & 6.5 & 7.0 & 6.5 & 6.75 & 5.0 \\
\hline 9 & 8.0 & 7.0 & 7.0 & 7.0 & 7.25 & 8.0 \\
\hline 10 & 7.0 & 6.0 & 6.0 & 7.0 & 6.5 & 5.0 \\
\hline 11 & 9.0 & 9.0 & 8.0 & 9.0 & 8.75 & 7.5 \\
\hline 12 & 9.0 & 8.0 & 8.0 & 9.0 & 8.5 & 8.0 \\
\hline 13 & 9.0 & 8.0 & 8.0 & 8.0 & 8.25 & 7.0 \\
\hline 14 & 9.0 & 8.0 & 7.0 & 8.0 & 8.0 & 7.0 \\
\hline 15 & 9.0 & 8.0 & 9.0 & 9.0 & 8.75 & 8.0 \\
\hline 16 & 9.0 & 8.0 & 9.0 & 8.0 & 8.5 & 7.5 \\
\hline 17 & 9.0 & 8.0 & 9.0 & 9.0 & 8.75 & 8.0 \\
\hline
\end{tabular}

Based on the obtained data, the numerical values of the regression coefficients for $Y_{1}$ and $Y_{2}$ were calculated and these regression equations can be presented as follows.

$$
\begin{gathered}
\mathrm{Y}_{1}=8.90+0.37 \cdot \mathrm{a}_{1}+0.11 \cdot \mathrm{a}_{2}+0.03 \cdot \mathrm{a}_{3}-1.05 \cdot \mathrm{a}_{11}-0.09 \cdot \mathrm{a}_{22}- \\
0.38 \cdot \mathrm{a}_{33}+0.04 \cdot \mathrm{a}_{12}-0.04 \cdot \mathrm{a}_{13}-0.09 \cdot \mathrm{a}_{23} \\
\mathrm{Y}_{2}=8.20+0.73 \cdot \mathrm{b}_{1}+0.16 \cdot \mathrm{b}_{2}+0.04 \cdot \mathrm{b}_{3}-0.98 \cdot \mathrm{b}_{11}-0.30 \cdot \mathrm{b}_{22}- \\
0.71 \cdot \mathrm{b}_{33}-0.06 \cdot \mathrm{b}_{12}-0.06 \cdot \mathrm{b}_{13}-0.06 \cdot \mathrm{b}_{23}
\end{gathered}
$$

During the optimization process, we determined the values of the influencing factors $X_{1}$ and $X_{2}$, which ensure the maximum of the response surface function.

The obtained values of the variables are (in dimensionless quantities): for CQC $\mathrm{X}_{1}=0.19, \mathrm{X}_{2}=0.63$, $\mathrm{X}_{3}=0.01$; for the function «Shelf life» $\mathrm{X}_{1}=0.36, \mathrm{X}_{2}=0.22$, $\mathrm{X}_{3}=-0.002$. When moving from dimensionless to real values, we get the optimal values of fermentation conditions: inoculate amount $(60 \pm 2) \mathrm{g} / \mathrm{l}$; fermentation time $(30 \pm 3) \mathrm{h}$; fermentation temperature $(35 \pm 4){ }^{\circ} \mathrm{C}$. Thus, carrying out the process under optimal conditions provides the highest value of CQC, which is 8.9 points, and the longest shelf life of the resulting fermented milk, equal to 8.2 days.

\subsection{Optimization of the recipe of fermented beverages with berry-juice}

On the base of fermented cow milk, in order to expand the range and improve the sensory characteristics, a certain amount of berry juice and sucrose was added. The main parameters influencing the quality indicators of fermented beverages with berry-juice, berry juice and sucrose amount, were varied at five levels. The number of experiments conducted, determined by the formula (2), was 9 points. The orthogonal planning matrix for 2 independent variables is presented in Table 5. 
Table 5. Matrix of the central composite design for optimizing the recipe of fermented beverages.

\begin{tabular}{|c|c|c|}
\hline \multirow[t]{3}{*}{ № } & \multicolumn{2}{|c|}{ The levels of factors } \\
\hline & Factor $X_{1}$ & Factor $\mathrm{X}_{2}$ \\
\hline & $\begin{array}{l}\text { Berry juice amount, } \\
\mathrm{g} / 100 \mathrm{~g} \text { of beverage }\end{array}$ & $\begin{array}{c}\text { Sucrose amount, g/100 } \\
\text { g of beverage }\end{array}$ \\
\hline 1 & 5 & 5 \\
\hline 2 & 15 & 5 \\
\hline 3 & 5 & 15 \\
\hline 4 & 15 & 15 \\
\hline 5 & 10 & 10 \\
\hline 6 & 15 & 10 \\
\hline 7 & 5 & 10 \\
\hline 8 & 10 & 15 \\
\hline 9 & 10 & 5 \\
\hline
\end{tabular}

The sensory properties of the described variants of the beverage were evaluated during laboratory tests, as described above in the section "Materials and methods". Comprehensive quality criterion (CQC) is a function of the response $\mathrm{Y}_{1}$.

After the sensory evaluation, the shelf-life time was determined and taken as the $\mathrm{Y}_{2}$ response function. The increase in $\mathrm{pH}$ during storage at a warm temperature is explained by the accumulation of microscopic fungi (molds, micromycetes) and putrefactive microorganisms, accompanied by enzymatic hydrolysis of milk proteins and followed by deamination and decarboxylation of amino acids produced. Since the solubility of the released ammonia is much higher than that of carbon dioxide, there is a pronounced leaching of the medium. The choice of temperature is determined by the operation convenience. At lower temperatures, the shelf life is up to 10-14 days, at higher temperatures- accelerates to 3-5 days.

Sensory indicators, CQC and shelf life of the fermented beverage with juices are shown in Tables 6-8.

Table 6. Sensory indicators, CQC and shelf life of a fermented beverage with sea buckthorn juice.

\begin{tabular}{|c|c|c|c|c|c|c|}
\hline № & $\mathrm{T}$ & $\mathrm{S}$ & $\mathrm{Cl}$ & $\mathrm{Cn}$ & $\mathrm{Y}_{1}$ & $\mathrm{Y}_{2}$ \\
\hline 1 & 8.0 & 7.0 & 7.0 & 6.0 & 7.0 & 6.0 \\
\hline 2 & 8.5 & 8.0 & 8.0 & 7.5 & 8.0 & 8.0 \\
\hline 3 & 8.0 & 7.5 & 7.5 & 8.0 & 7.7 & 6.25 \\
\hline 4 & 8.0 & 8.0 & 8.5 & 7.5 & 8.0 & 7.5 \\
\hline 5 & 8.5 & 8.0 & 8.0 & 8.0 & 8.2 & 8.0 \\
\hline 6 & 8.5 & 9.0 & 8.5 & 8.5 & 8.75 & 8.5 \\
\hline 7 & 8.0 & 7.0 & 8.0 & 8.0 & 7.75 & 6.0 \\
\hline 8 & 8.0 & 8.5 & 7.0 & 7.5 & 8.0 & 8.2 \\
\hline 9 & 8.0 & 7.0 & 8.0 & 8.0 & 7.75 & 7.0 \\
\hline
\end{tabular}

Table 7. Sensory indicators, CQC and shelf life of a fermented beverage with chokeberry juice.

\begin{tabular}{|c|c|c|c|c|c|c|}
\hline № & $\mathrm{T}$ & $\mathrm{S}$ & $\mathrm{Cl}$ & $\mathrm{Cn}$ & $\mathrm{Y}_{1}$ & $\mathrm{Y}_{2}$ \\
\hline 1 & 8.0 & 7.0 & 7.5 & 6.0 & 7.2 & 6.2 \\
\hline 2 & 8.5 & 8.0 & 8.5 & 7.5 & 8.2 & 8.2 \\
\hline 3 & 8.5 & 7.0 & 7.5 & 8.0 & 7.7 & 6.5 \\
\hline 4 & 8.5 & 8.0 & 8.5 & 7.5 & 8.2 & 7.75 \\
\hline 5 & 8.5 & 8.0 & 8.0 & 7.5 & 8.0 & 8.0 \\
\hline 6 & 8.5 & 8.0 & 9.0 & 7.5 & 8.5 & 8.7 \\
\hline 7 & 8.0 & 7.0 & 7.5 & 8.0 & 7.25 & 6.2 \\
\hline 8 & 7.0 & 8.5 & 8.0 & 7.5 & 8.0 & 8.0 \\
\hline 9 & 8.0 & 8.0 & 8.0 & 7.0 & 7.75 & 6.75 \\
\hline
\end{tabular}

Table 8. Sensory indicators, CQC and shelf life of a fermented beverage with lingonberry juice.

\begin{tabular}{|c|c|c|c|c|c|c|}
\hline № & $\mathrm{T}$ & $\mathrm{S}$ & $\mathrm{Cl}$ & $\mathrm{Cn}$ & $\mathrm{Y}_{1}$ & $\mathrm{Y}_{2}$ \\
\hline 1 & 8.0 & 7.5 & 7.5 & 7.0 & 7.5 & 6.2 \\
\hline 2 & 8.5 & 8.0 & 7.5 & 7.5 & 8.0 & 8.0 \\
\hline 3 & 8.5 & 7.0 & 7.5 & 7.0 & 7.5 & 6.5 \\
\hline 4 & 8.5 & 8.0 & 8.5 & 7.0 & 8.0 & 7.7 \\
\hline 5 & 8.5 & 8.0 & 8.0 & 8.0 & 8.12 & 7.75 \\
\hline 6 & 9.0 & 8.0 & 9.0 & 7.5 & 8.75 & 8.5 \\
\hline 7 & 8.0 & 7.0 & 7.5 & 7.0 & 7.0 & 6.25 \\
\hline 8 & 7.0 & 8.0 & 8.0 & 7.0 & 7.5 & 8.0 \\
\hline 9 & 9.0 & 8.0 & 8.0 & 7.0 & 7.0 & 7.5 \\
\hline
\end{tabular}

Based on the obtained data, the values of the regression coefficients for $Y_{1}$ and $Y_{2}$ were calculated, and these regression equations are as follows.

The beverage with sea buckthorn juice:

$$
\begin{aligned}
& Y_{1}=9.56+0.38 \cdot a_{1}+0.15 \cdot a_{2}+0.45 \cdot a_{11}+0.06 \cdot a_{22}-0.18 \cdot a_{12} \\
& Y_{2}=7.19+0.96 \cdot b_{1}+0,16 \cdot b_{2}+0.02 \cdot b_{11}+0.10 \cdot b_{22}-0.18 \cdot b_{12}
\end{aligned}
$$

With chokeberry juice:

$$
\begin{aligned}
& Y_{1}=10.53+0.46 \cdot a_{1}+0.13 \cdot a_{2}+0.45 \cdot a_{11}+0.06 \cdot a_{22}-0.13 \cdot a_{12} \\
& Y_{2}=7.28+0.96 \cdot b_{1}+0.18 \cdot b_{2}+0.02 \cdot b_{11}+0.10 \cdot b_{22}-0.19 \cdot b_{12}
\end{aligned}
$$

With lingonberry juice:

$$
\mathrm{Y}_{1}=9.42+0.46 \cdot \mathrm{a}_{1}+0.45 \cdot \mathrm{a}_{11}+0.06 \cdot \mathrm{a}_{22}-0.02 \cdot \mathrm{a}_{12}
$$

$$
\mathrm{Y}_{2}=7.21+0.87 \cdot \mathrm{b}_{1}+0.20 \cdot \mathrm{b}_{2}+0.02 \cdot \mathrm{b}_{11}+0.10 \cdot \mathrm{b}_{22}-0.15 \cdot \mathrm{b}_{12}
$$

During the optimization process, we determined the numerical values of the influencing factors $X_{1}$ and $X_{2}$, which ensure the maximum of the response surface function.

The obtained numerical values of the variables in dimensionless quantities are as follows.

For the CQC function with sea buckthorn $\mathrm{X}_{1}=0.72$, $\mathrm{X}_{2}=0.48$, chokeberry $\mathrm{X}_{1}=0.84, \mathrm{X}_{2}=0.65$, lingonberry $\mathrm{X}_{1}=0.67, \mathrm{X}_{2}=0.56$.

For the function "Shelf life" with sea buckthorn $\mathrm{X}_{1}=0.85, \quad \mathrm{X}_{2}=0.02, \quad$ chokeberry $\mathrm{X}_{1}=0.78, \quad \mathrm{X}_{2}=0.06$, lingonberry $\mathrm{X}_{1}=0.8, \mathrm{X}_{2}=0.1$.

In natural values, the optimal recipes of fermented beverages are: $12.2 \mathrm{~g}$ of sea buckthorn juice and $9.3 \mathrm{~g}$ of sucrose per $100 \mathrm{ml}$ of beverage; $13.131 \mathrm{~g}$ of chokeberry juice and $11.3 \mathrm{~g}$ of sucrose per $100 \mathrm{ml}$ of beverage; $11.632 \mathrm{~g}$ of lingonberry juice and $10.1 \mathrm{~g}$ of sucrose per $100 \mathrm{ml}$ of beverage.

\section{Conclusions}

Using the method of Central Composite Design (CCD), we optimized the conditions for milk fermentation by Tibetan kefir grains. The numerical values of the influencing factors are: the dose of the introduced starter culture is $(60 \pm 2) \mathrm{g}$ per $100 \mathrm{ml}$ of milk; fermentation time is $(30 \pm 3) \mathrm{h}$; fermentation temperature is $(35 \pm 4){ }^{\circ} \mathrm{C}$.

The effect of berry juices on the quality indicators of fermented milk beverages and on their shelf life was studied. 
The second-order dependencies of complex quality criterion (CQC) and shelf life of fermented beverages with berry juices on the component content of the beverage were calculated. According to the results, the recipe of the fermented beverage based on the Tibetan kefir grains was modified. The optimal components content (per $100 \mathrm{ml}$ of the beverage) is: sea buckthorn, chokeberry and lingonberry juice - $(12 \pm 2) \mathrm{g}$, sucrose$(10 \pm 1) \mathrm{g}$.

\section{References}

1. N. Sanlier, B.B. Gökcen, A.C. Sezgin, Crit Rev. Food Sci Nutr., 59, 506-527 (2019)

2. Z.F. Bhat, H. Bhat, Int. J. of Dairy Sci., 6 (1), 1$12(2011)$

3. V.K. Shiby, H.N. Mishra., Crit Rev Food Sci Nutr., 53 (5), 482-496 (2013)

4. S.N. Casarotti, D.A. Monteiro, M.S. Moretti, A.L.B. Penna, Food Res. Int., 59, 67-75 (2014)

5. L.M. Beltrán-Barrientos, A. Hernández-Mendoza, M.J. Torres-Llanez, A.F. González-Córdova, B. Vallejo-Córdoba, J. Dairy Sci., 99 (6), 4099-4110 (2016)

6. N. Yamamoto, Bioactive Proteins and Peptides as Functional Foods and Nutraceuticals (eds Y. Mine, E. Li Chan and B. Jiang) (2010)

7. N. Yamamoto, Biopolymers, 43 (2), 129-134 (1997)

8. K. Inoue, T. Gotou, H. Kitajima, S. Mizuno, T. Nakazawa, N. Yamamoto, J. Biosci. Bioeng., 108 (2), 111-115 (2009)

9. N. Yamamoto, M. Maeno, T. Takano, J. Dairy Sci., 82 (7), 1388-1393 (1999)

10. N. Yamamoto, A. Akino, T. Takano, Biosci., Biotech. and Biochem., 58 (4), 776-778 (1994)

11. S. Otles, O. Cagindi, Pakist. J. Nutr., 2 (2), 54-59 (2003)

12. M.R. Prado, L.M. Blandón, L.P.S. Vandenberghe, C. Rodrigues, G.R. Castro, V. Thomaz-Soccol, C.R. Soccol, Front. Microbiol., 6, 1177 (2015)

13. A. Fardet, E. Rock, Nutr Res Rev., 31 (1), 52-70 (2018)

14. W. Tang, C. Li, Z. He, F. Pan, S. Pan, Y. Wang, Probiotics \& Antimicrob. Prot., 10, 523-533 (2018)

15. Y. Zheng, Y. Lu, J. Wang, L. Yang, C. Pan, Y. Huang, PLOS One, 8, 7, e698682013

16. Y. Huang, F. Wu, X. Wang, Y. Sui, L. Yang, J. Wang, J. Dairy Sci., 96, 2816-2825 (2013)

17. J. Zhang, X. Zhao, Y. Jiang, W. Zhao, T. Guo, Y. Cao, J. Teng, X. Hao, J. Zhao, Z. Yang J. Dairy Sci., 100 (8), 6025-6041 (2017)

18. O.M. Tikhomirova, E.A. Ivanova, Problems in medical mycology (rus.), 17, 58-62 (2015)

19. J. Mei, Q. Guo, Y. Wu, Y. Li, H. Yu, Food Sci. Biotechnol., 24 (2), 393-402 (2015)

20. J. Mei, Q. Guo, Y. Wu, Y. Li, PLoS ONE, 9 (10), e111648 (2014)

21. J. Mei, F. Feng, Q. Guo, Y. Li, Y. Wu, Food Sci. Biotechnol., 24 (3), 1017-1027 (2015)
22. B. Li, X. Gao, N. Li, J. Mei, Czech J. Food Sci., 36 (6), 494-501 (2018)

23. K.J. Heller, Am. J. Clin Nutr., 73 (2 Suppl), 374379 (2001)

24. J. Gao, F. Gu, H. Ruan, Q. Chen, J. He, G. He, Proc. Engineer., 37, 132-136 (2012)

25. M. Slozhenkina, I. Gorlov, V. Kryuchkova, N. Mosolova, A. Bochkareva, O. Serova, IOP Conf. Ser. Earth Environ. Sci., 548, 082030 (2020)

26. O.K. Gogaev, D.G. Morgoeva, A.R. Demurova, Z.A. Karaeva, G.S. Tukfatulin, R.S. Godzhiev, E.A. Tokhtieva, L.O. Gogaeva, Indo Amer. J. Pharm. Sci., 6 (8), 14873-14880 (2019)

27. O. Kochubei-Lytvynenko, I. Korolchuk, U. Kuzmyk, N. Frolova, V. Pasichny, N. Yushchenko, I. Mykoliv, Ukr. J. Food Sci., 7, 2 (2019)

28. I.V. Smotrayeva, P.E. Balanov, O.B. Ivanchenko, R.E. Khabibulin, Bull. Kazan techn. Univers. (rus.), 17 (22), 229-231 (2014)

29. A.V. Sokolova, O.B. Ivanchenko, R.E. Khabibullin, Bull. Kazan techn. Univers. (rus.), 19 (24), 157-159 (2016)

30. S.S. Nielsen, Food Analysis, Food Science Texts Series (Springer Science+Business Media, LLC 2010, 602)

31. S. Redha, I. Muiz, J. Glob. Pharm.Techn., 10, 7 (2018). 